\title{
BMJ Open Quality Using quality improvement to improve the utilisation of GeneXpert testing at five lab hubs in Northern Uganda
}

\author{
Esther Karamagi, ${ }^{1}$ Joseph Nturo, ${ }^{1}$ Pamela Donggo, ${ }^{1}$ Isabel Kyobutungi, ${ }^{1}$ \\ Judith Aloyo, ${ }^{1}$ Simon Sensalire, ${ }^{1}$ Mirwais Rahimzai ${ }^{2}$
}

To cite: Karamagi E, Nturo J, Donggo $\mathrm{P}$, et al. Using quality improvement to improve the utilisation of GeneXpert testing at five lab hubs in Northern Uganda.BMJ Open Quality 2017;6:e000201. doi:10.1136/ bmjoq-2017-000201

Received 11 September 2017 Revised 14 November 2017 Accepted 21 November 2017

\section{CrossMark}

${ }^{1}$ USAID Applying Science to Strengthen and Improve Systems (ASSIST) Project, University Research Co, LLC Kampala, Uganda ${ }^{2}$ USAID Applying Science to Strengthen and Improve Systems (ASSIST) Project, University Research Co, LLC Chevy Chase, Maryland, USA

Correspondence to Dr Esther Karamagi; ekaramagi@urc-chs.com

\section{BACKGROUND}

Tuberculosis (TB) continues to be a major public health problem, with an estimated 10.4 million new (incident) TB cases worldwide each year. ${ }^{1}$ To reduce the burden of TB disease, case detection and treatment gaps should be addressed to interrupt transmission chains and reduce individual morbidity. Sputum smear microscopy, the most widely used test for diagnosing $\mathrm{TB}$, has a sensitivity of only $50 \%$ among active TB cases and contributes to a delay in diagnosis and continued transmission. Sputum smears with chest X-ray, where available, are the tests routinely applied for TB diagnosis. However, the inaccessibility and cost of X-ray diagnosis make it prohibitive for rural settings like Northern Uganda. The GeneXpert machine is preferred because it requires less expertise, is more sensitive than microscopy and can detect multidrug-resistant TB. ${ }^{2}$

Case notification for TB in the 16 districts in Northern Uganda is 134 cases/100 000 population, well below the national target of 161/100 000. ${ }^{3}$ Improved TB diagnostics in this region is critical to attain timely case detection and management of $\mathrm{TB}$, and reduce mortality, transmission and prevalence of the disease.

\section{OBJECTIVE}

With the aim of increasing the number of GeneXpert samples processed, the US Agency for International Development Applying Science to Improve Systems (ASSIST) Project began working to improve GeneXpert services at five laboratory hubs in the Northern Uganda region in March 2016: Kitgum, Anaka and Apac hospitals, and Amolatar and Madi-opei health centre IVs. All five GeneXpert machines can run up to four samples every 2 hours, yet weekly data showed that about five samples were being run each day. In all, only 91 samples had been processed per month using GeneXpert machines instead of a maximum of about 1600 samples per month with eight working hours per day, hence an underutilisation of the machines.

\section{METHODS FOR IMPROVEMENT}

Scaling up the use of GeneXpert in the region did not require increasing the number of machines procured and installed, but rather using the current machines to their optimal capacity. In March 2016, ASSIST together with Ministry of Health teams conducted a GeneXpert utilisation assessment in the northern region and revealed the following gaps across the five GeneXpert sites in the region:

1. A lot of missed opportunities for eligible patients to test were created because facilities were too stringent in making presumed patients with TB provide early morning samples (often inconvenient for patients) instead of spot samples.

2. Data about processed samples were documented inconsistently in the different registers, which meant that the facilities had no clear way of tracking samples processed by the GeneXpert machines.

3. There were interruptions of operations due to electricity outages.

4. All hubs reported stock out of cartridges.

5. There was a lack of monitoring of stock distribution and use of GeneXpert at the facility level.

In March 2016, the findings of the assessment were discussed and shared data with the existing facility quality improvement (QI) teams, which comprised laboratory personnel, staff from the HIV clinic, clinicians and TB nurses who were focused on improving processes and outcomes of people with HIV and TB for purposes of sustainability. The QI teams then carried out site-specific root cause analyses of the low numbers of samples 


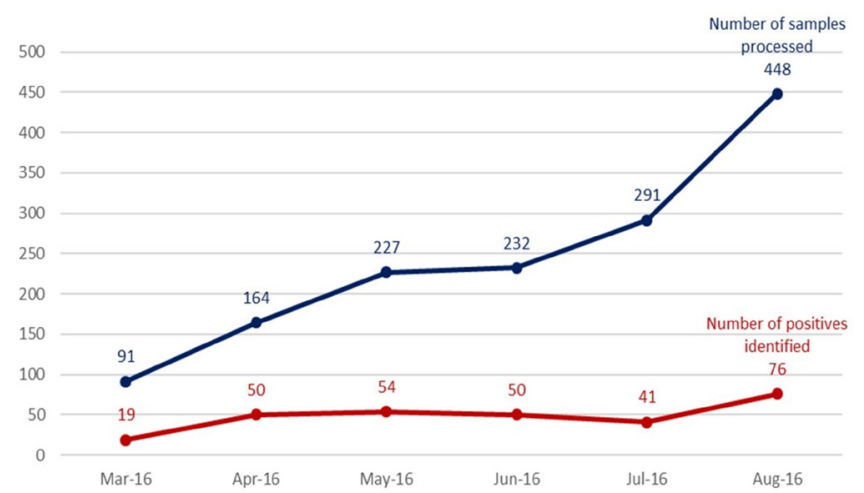

Figure 1 Number of GeneXpert samples processed and number of tuberculosis-positive cases identified in five sites, Uganda (March 2016-August 2016).

processed by the GeneXpert machines. Three root causes were identified: (1) almost no samples were referred from facilities outside of the GeneXpert site; (2) many samples tested by the GeneXpert site were not reported; and (3) samples from within the GeneXpert site were few, compared with the number of eligible patients managed by each site. The teams planned and tested several changes using Plan-Do-Study-Act cycles to improve the number of samples tested from an average of four samples per site per day to the target of 16 samples. These included the following: (1) To address the problem of referrals, routes of hub riders who were responsible for picking samples from referral sites were rescheduled. The referral sites also started using triple packaging of samples as opposed to single packaging. These changes avoided expiration and leakage of samples from referral sites, which was previously leading to sample rejection and demotivation of the referring team. (2) To address reporting, a GeneXpert focal person was appointed per facility and added to the QI team. This person was responsible for checking that all GeneXpert samples were tested and reported to the National TB and Leprosy Laboratory or to ASSIST and that data were entered into the electronic reporting system. For continuity, in September 2016, ASSIST provided internet modems to the focal persons, who then started entering the data directly into the system weekly. (3) To address the problem of few samples being tested at site level, the team introduced GeneXpert testing for all eligible HIV-positive patients. The team also addressed logistics problems, such as timely ordering of GeneXpert cartridges, to ensure they did not run out of cartridges to test all samples.

\section{RESULTS}

Following the implementation of these changes between March and August 2016, the teams achieved an increase in the number of samples processed from 91 to 448 , and the number of identified TB-positives from 19 to 76 in the five sites (figure 1). However, this is a proxy and not sufficient evidence of the trend on TB case notification.
The five laboratories were able to absorb the additional numbers.

\section{CONCLUSION}

This experience points to how process improvement in healthcare facilities can improve TB case detection without additional resources and in a short time by optimising existing use of GeneXpert machines. We learnt that an effective and efficient hub transportation and sample collection system will generate more TB samples. However, with more samples, improved logistics are needed for testing and reporting. The project therefore supported using the Uganda National Health Laboratory hotline to request and report stock status for GeneXpert cartridges. We also supported interhub referrals of samples for the sites that experienced breakdown of their GeneXpert machines. To ensure monitoring of the data from the GeneXpert machines, a GeneXpert alert system was installed in the machines and internet modems fitted for transmitting data from the GeneXpert machine, through the alert system, to the National Tuberculosis Reference Laboratory. Since improvement techniques were tested and deployed within routine health systems structures, these structures can be supported to sustain the observed improvement.

Acknowledgements The authors acknowledge district health officers and health facility staff especially the laboratory personnel in the intervention sites.

\section{Collaborators Dr Alex Muhereza, Dr Esther Ochora.}

Contributors All authors have made substantive intellectual contributions to this paper: EK and MR conceived the intervention. JA, JN, PD, IK and SS planned, rolled out the intervention and collected data. SS analysed the data and drafted the paper with EK and JA. All authors critically appraised, reviewed and approved the final version of the paper.

Funding The intervention described in this paper was made possible by the support of the American people through US President's Emergency Plan for AIDS Relief (PEPFAR) and the US Agency for International Development (USAID), through the USAID Applying Science to Strengthen and Improve Systems (ASSIST) Project, implemented by University Research Co, LLC under Cooperative Agreement Number AID-OAA-A-12-00101.

Competing interests None declared.

Provenance and peer review Not commissioned; externally peer reviewed.

Open Access This is an Open Access article distributed in accordance with the Creative Commons Attribution Non Commercial (CC BY-NC 4.0) license, which permits others to distribute, remix, adapt, build upon this work non-commercially, and license their derivative works on different terms, provided the original work is properly cited and the use is non-commercial. See: http://creativecommons.org/ licenses/by-nc/4.0/

C) Published by the BMJ Publishing Group Limited. For permission to use (where not already granted under a licence) please go to http://www.bmj.com/company/ products-services/rights-and-licensing/

\section{REFERENCES}

1. World Health Organization. Global Tuberculosis Report, 2016. Geneva: WHO, 2016.

2. Lawn SD, Nicol MP. Xpert ${ }^{\circledR}$ MTB/RIF assay: development, evaluation and implementation of a new rapid molecular diagnostic for tuberculosis and rifampicin resistance. Future Microbiol 2011;6:1067-82.

3. Uganda Ministry of Health. Annual health sector performance report, 2015/2016. Kampala, Uganda: Uganda Ministry of Health, 2016. 